\title{
Importance of Basset History Force for the Description of Magneti- cally Driven Motion of Magnetic Particles in Air
}

\author{
Andrej Krafcik ${ }^{1}$, Peter Babinec ${ }^{2}$, Melania Babincova ${ }^{2}$, Ivan Frollo ${ }^{1}$ \\ ${ }^{1}$ Department of Imaging Methods, Institute of Measurement Science, Slovak Academy of Sciences, Dubravska cesta 9, 84104, \\ Bratislava, Slovakia, andrej.krafcik@savba.sk \\ ${ }^{2}$ Department of Nuclear Physics and Biophysics, Faculty of Mathematics, Physics and Informatics, Comenius University, \\ Mlynska dolina F1, 84248 Bratislava, Slovakia
}

\begin{abstract}
Lungs are used as an attractive possibility for administration of different therapeutic substances for a long time. An innovative method of such administration widely studied nowadays is the application of aerosolized magnetic particles as the carriers to the lungs in the external non-homogeneous magnetic field. For these reasons we have studied dynamics of such a system on a level of particle trajectory in air in the presence of magnetic force as a driving force exerted on micrometric magnetic particle. On two typical examples of magnetically driven systems - motion of magnetic particle in a gradient magnetic field and cyclotron-like motion of a charged particle in homogeneous magnetic field in microscale, where the external accelerating forces are very large and the relevant time scale is of the order from fraction of milliseconds to seconds, we have examined the importance of these forces. As has been shown, for particles with high initial acceleration, not only the commonly used Stokes force but also the Basset history force should be used for correct description of the motion.
\end{abstract}

Keywords: Aerosol, magnetically driven particle motion, Stokes force, Basset history force, Maxey-Riley equation, numerical simulation.

\section{INTRODUCTION}

Computational simulation, as an important tool for the study of fluid dynamics in biophysics and biotechnology, allows to quickly improve the device functionality, minimizing the cost of prototype testing, or can help to describe systems unreachable experimentally [1], [2], [3], [4], [5], [6], [7].

In the Lagrangian simulation frame mostly used in models, the viscous Stokes' drag force is usually concerned as a dominant. In 1888 A. B. Basset [8] showed that the governing equation of motion for small spherical inertial particles should be formulated as an integro-differential equation with integral term denoted as the Basset history force, which accounts for the diffusion of vorticity around the particle throughout its entire history.

Recent experimental and numerical studies, however, show that this history term influences the dynamics of inertial particles significantly and therefore cannot be generally neglected [9], [10], [11], [12], [13], [14]. For flow across an aerodynamic shock, the Basset history force acting upon the particle described by the Basset integral can be substatialy larger than the viscous drag in the immediate shock region [15]. Also the effects of the history force on an oscillating rigid sphere at low Reynolds number cannot be neglected [16]. Particlefluid systems can be treated as aerosols or bubbles depending on a ratio of their mass densities. Garbin et al. [17] studied

DOI: $10.2478 / \mathrm{msr}-2020-0007$ history force influence on microbubbles propelled by ultrasound, and showed that the instantaneous values of hydrodynamic forces extracted from experimental data confirm that the history force accounts for the largest part of the viscous force. For an impulsively started flow over a bubble, accurate finite difference results show that the history force on the bubble decays as $t^{-2}$ at large time [18]. Another interesting observation is the influence of the Basset history force on the change of both the nature and the number of attractors of inertial particle advection for gravitational effects and horizontal diffusion [10]. Recently, it was also shown that the Basset history force has a negligible effect on linear stability of particle laden flows [19]. History force affects chaotic advection of inertial particles in a rather non-trivial way as has been shown in [11]. A recent simulation [20] has shown reduction effect of small-scale clustering of inertial particles transported in homogeneous and isotropic turbulence. As we have recently shown, the Basset history force is important also for correct description of high gradient magnetic separation [21]. Basset force, due to its difficult computation, is often neglected. Nevertheless, recently there were efforts to develop an efficient integral/quadrature scheme for its numerical evaluation [22], [23], [24], [25] as summarized in [26].

Lungs are an attractive interface for administration of various therapeutic substances for common diseases, e.g., delivery of insulin [27]. Posibility of using magnetic carriers has 
been recently studied on experimental, as well as theoretical basis [7], [28], [29], [30], [31], in order to increase the efficiency of this pulmonary route. Nevertheless, the Basset history force is not taken into account. It is therefore our aim in this study to implement a model describing the motion of a micrometric sized spherical magnetic particle in air as quiescent fluid ambient for comparison of the importance of all relevant forces describing the dynamics of such sphere in two widely used examples of magnetic systems - motion of a sphere with magnetic moment in a gradient magnetic field and cyclotron-like motion of a charged sphere in homogeneous magnetic field.

\section{MATERIAL AND METHODS}

\subsection{Physical model}

\subsubsection{Governing equations}

The governing equation for the movement of spherical body in viscous fluid ambient was derived already at the end of the $19^{\text {th }}$ century by A. B. Basset [8] as a classic theoretical result from the unsteady Stokes' equation, further developed by Boussinesq and Oseen, and nowadays used in the generalized Maxey-Riley form [11], [32] as

$$
\begin{aligned}
m_{\mathrm{p}} \frac{\mathrm{d} \vec{v}}{\mathrm{~d} t} & =m_{\mathrm{f}} \frac{\mathrm{D} \vec{u}}{\mathrm{D} t}-\frac{m_{\mathrm{f}}}{2}\left(\frac{\mathrm{d} \vec{v}}{\mathrm{~d} t}-\frac{\mathrm{D} \vec{u}}{\mathrm{D} t}\right) \\
& -6 \pi b \rho_{\mathrm{f}} v(\vec{v}-\vec{u})+\left(m_{\mathrm{p}}-m_{\mathrm{f}}\right) \vec{g} \\
& -6 b^{2} \rho_{\mathrm{f}} \sqrt{\pi v} \int_{t_{0}}^{t} \mathrm{~d} \tau \frac{1}{\sqrt{t-\tau}}\left(\frac{\mathrm{d} \vec{v}}{\mathrm{~d} \tau}-\frac{\mathrm{d} \vec{u}}{\mathrm{~d} \tau}\right) \\
& +\vec{F}_{\text {Mag }},
\end{aligned}
$$

where $m_{\mathrm{p}}$ and $m_{\mathrm{f}}$ are the mass of the particle and mass of the fluid excluded by the particle, respectively; $\vec{v} \equiv \mathrm{d} \vec{r} / \mathrm{d} t$ is the particle velocity, $\vec{u}(\vec{r}, t)$ is the velocity vector field of fluid ambient; and

$$
\begin{aligned}
\frac{\mathrm{d} \vec{u}}{\mathrm{~d} t} & \equiv \frac{\partial \vec{u}}{\partial t}+\vec{v} \cdot \vec{\nabla} \vec{u}, \\
\frac{\mathrm{D} \vec{u}}{\mathrm{D} t} & \equiv \frac{\partial \vec{u}}{\partial t}+\vec{u} \cdot \vec{\nabla} \vec{u},
\end{aligned}
$$

denote the full derivative along the trajectory of the particle and of the corresponding fluid element, respectively. The remaining symbols in (1): $b, \rho_{\mathrm{f}}$, and $v$ are spherical particle radius, and density, and kinematic viscosity of fluid, respectively. The terms on the right-hand-side of (1) are identified as: the force exerted by the fluid on a fluid element at the location of the particle (further denoted as the fluid force), the added mass term describing the impulsive pressure response of the fluid, the Stokes drag, the buoyancy-reduced gravity, the Basset history force term (an integral accounts for the viscous diffusion of vorticity from the surface of the particle along its trajectory) [11], and the external magnetic force term exerting on particle.

Integral in the Basset history force term can be put in the case of non-zero initial difference of particle and fluid element velocity to the form [11], [33]

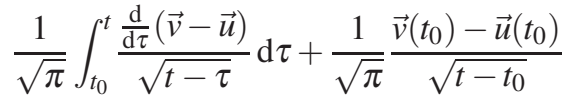

$$
=\frac{1}{\sqrt{\pi}} \frac{\mathrm{d}}{\mathrm{d} t} \int_{t_{0}}^{t} \frac{\vec{v}-\vec{u}}{\sqrt{t-\tau}} \mathrm{d} \tau
$$

using integration by parts.

Equation (1) involving (4) can be rewritten to the dimensionless form after using characteristic time $T$, velocity $U$, and length $a$ scales explained later:

$$
\begin{aligned}
\frac{1}{R} \frac{\mathrm{d} \overrightarrow{\hat{v}}}{\mathrm{~d} \hat{t}} & =\frac{3}{2} \frac{\mathrm{D} \overrightarrow{\hat{u}}}{\mathrm{D} \hat{t}}-\frac{1}{S}(\overrightarrow{\hat{v}}-\overrightarrow{\hat{u}}-W \vec{n}) \\
& -\sqrt{\frac{9}{2 \pi} \frac{1}{S}} \frac{\mathrm{d}}{\mathrm{d} \hat{t}}\left[\int_{\hat{t}_{0}}^{\hat{t}} \frac{\overrightarrow{\hat{v}}-\overrightarrow{\hat{u}}}{\sqrt{\hat{t}-\tau}} \mathrm{d} \tau-\frac{\overrightarrow{\hat{v}}\left(\hat{t}_{0}\right)-\overrightarrow{\hat{u}}\left(\hat{t}_{0}\right)}{\sqrt{\hat{t}-\hat{t}_{0}}}\right] \\
& +\overrightarrow{\hat{F}}_{\mathrm{Mag}},
\end{aligned}
$$

where the hat over the variable denotes its dimensionless form, $\vec{n}$ is a unit vector pointing upwards against gravity, and the three dimensionless parameters used:

$$
\begin{aligned}
R & =\frac{2 \rho_{\mathrm{f}}}{\rho_{\mathrm{f}}+2 \rho_{\mathrm{p}}}, \\
S & =\frac{2}{9} \frac{b^{2} / v}{T}, \\
W & =S\left(\frac{3}{2}-\frac{1}{R}\right) \frac{g T}{U},
\end{aligned}
$$

are the density parameter, the size parameter, and the dimensionless settling velocity, respectively [11]. The $\rho_{\mathrm{p}}$ denotes the particle mass density.

\subsubsection{Magnetic force}

The magnetic force term used in our simulations depends on the studied problem. We are analyzing two common problems:

1. the magnetic force exerting on superparamagnetic spherical particle with magnetic susceptibility $\chi$ moving in external gradient magnetic field [34] of single circular current loop with magnetic flux density $\vec{B}$ :

$$
\vec{F}_{\text {Mag }}=\chi V_{\mathrm{p}} \vec{\nabla}\left(\frac{1}{2} \vec{H} \cdot \vec{B}\right)
$$

can be expressed (using relation $\vec{H}=\vec{B} / \mu_{0}$ ) as its $i$-th component in dimensionless form as

$$
\hat{F}_{\mathrm{Mag}, i}=\frac{1}{S} \frac{v_{\mathrm{m}}}{U}\left(\hat{B}_{j} \hat{\partial}_{i} \hat{B}_{j}\right),
$$

where the Einstein summation convention was used and $v_{\mathrm{m}}=2 b^{2} \chi \mu_{0} I^{2} / 9 \pi^{2} v \rho_{\mathrm{f}} a^{3}$ is the 'magnetic velocity', with radius of current loop and current magnitude $a$ and $I$, respectively, and external magnetic flux density expressed in dimensionless form as $\overrightarrow{\hat{B}} \equiv \vec{B} a \pi / \mu_{0} I$ (general expression for $\vec{B}$ or its gradient using elliptic integrals can be found in [35] or in shortened version in A). The $V_{\mathrm{p}}$ and $\hat{\partial}_{i}$ are the particle volume and $i$-th component of dimensionless partial derivative operator, respectively. 
2. the magnetic force on moving particle in cyclotron-like homogeneous magnetic field with magnetic flux density $\vec{B} \equiv\left(0,0, B_{0}\right)$ and particle carrying electric charge $q$ moving with non-zero initial velocity $\vec{v}(0) \equiv(0, \omega a, 0)$ : $\vec{F}_{\text {Mag }}=q(\vec{v} \times \vec{B})=m_{\mathrm{p}}(\vec{\omega} \times \vec{v})$ can be expressed in dimensionless form as

$$
\vec{F}_{\mathrm{Mag}}=\frac{1}{S} \frac{v_{\mathrm{m}}}{U}(\overrightarrow{\hat{\omega}} \times \overrightarrow{\hat{v}}),
$$

where $v_{\mathrm{m}}=2 b^{2} \rho_{\mathrm{p}} U \omega / 9 v \rho_{\mathrm{f}}$ is the 'magnetic velocity ', $a$ is initial radial distance of particle from axis, the angular velocity vector is defined as

$$
\vec{\omega}=-q \vec{B} / m_{\mathrm{p}} \equiv(0,0, \omega)
$$

and its dimensionless form as $\overrightarrow{\hat{\omega}}=\vec{\omega} / \omega$.

\subsubsection{Quiescent fluid ambient}

In both modeled problems we have considered as a fluid quiescent air ambient at $300 \mathrm{~K}$. Therefore, the total derivative along the trajectory of the fluid element corresponding to the particle (3) is from definition zero, or explicitly

$$
\frac{\mathrm{D} \vec{u}}{\mathrm{D} t} \equiv \frac{\partial \vec{u}}{\partial t}+\vec{u} \cdot \vec{\nabla} \vec{u}=\overrightarrow{0}
$$

and the force exerted by the fluid on a fluid element at the location of particle also diminishes.

\subsection{Numerical integration of the Maxey-Riley equation}

The Maxey-Riley equation (1) or its dimensionless form (5) is an integro-differential equation, which means that during the integration process in each time step it is necessary to compute the Basset history force integral term. For this purpose we used variable time step order one quadrature scheme presented in [36] proposed in [25]. In this sense, the MaxeyRiley equation (5) can be transcribed to the system of ordinary differential equations (ODEs). Moreover, using variable time step definition of quadrature scheme allowed us to use standard collection of MATLAB ${ }^{\mathrm{TM}}$ (The MathWorks, 2017) ODEs solvers for their calculation. For this purpose we have used as the most suitable solver ode $15 \mathrm{~s}$, based on the numerical differential formulas of orders 1 to 5 [37].

Numerical calculations of studied problems were governed by error manipulation, in particular by absolute error tolerances: $\mathrm{AbsTol}=10^{-12}$ for dimensionless location variables, Abs Tol $=10^{-10}$ for dimensionless velocity variables, and with $\operatorname{RelTol}=10^{-12}$ as the relative error tolerance for both dimensionless location and velocity variables.

\section{RESULTS AND DISCUSSION}

We have performed simulations for MyOne ${ }^{\mathrm{TM}}[38]$ widely used one micron bead particle in quiescent air as fluid ambient for two modeled problems: a) magnetic particle in the gradient magnetic field of single circular current loop with radius $a$ and current magnitude $I$ located in $x y$-plane with axis identical with $z$-axis of the Cartesian coordinate system and with initial position $\vec{r}(0)=(-1 / 3 a, 0,-a)$ and zero initial velocity (Fig.1.), and b) particle in cyclotron-like homogeneous magnetic field moving with angular velocity vector $\vec{\omega}$ in perpendicular plane to the homogeneous magnetic field with initial radial distance $a$ and initial velocity $U$.

(a)
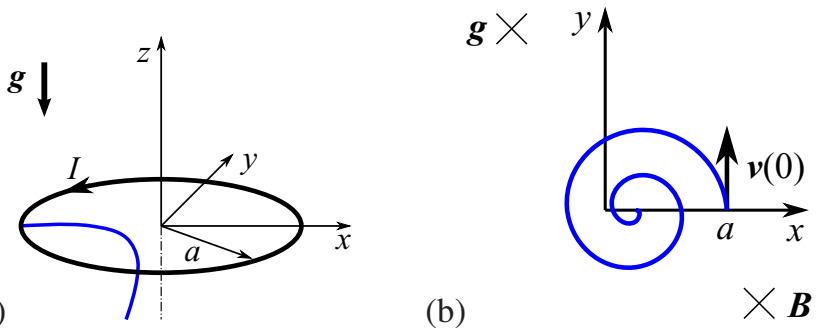

Fig.1. Schematic diagram (a) of single circular current loop arrangement; and (b) arrangement of particle in cyclotron-like magnetic field.

For both studied problems we have analyzed two subproblems: (i) a movement of particle described with the Newton equation of motion with magnetic force and buoyancyreduced gravity as driving forces and the Stokes' viscous drag as a single drag force; and (ii) the movement of particle described with the Maxey-Riley equation (section 2.1) combining together with the three mentioned forces in previous subproblem also fluid, added mass, and the Basset history force. The fluid force was due to quiescentness of air as fluid ambient neglected. The first and the second sub-problems were further denoted for abbreviation as the Stokes and the Basset problem, respectively.

Values of parameters used in simulations are shown in Table 1 .

\subsection{Magnetic particle in gradient magnetic field of circular current loop}

In Fig.2. are shown trajectories of magnetic particle in the magnetic field of circular current loop in air as quiescent fluid ambient for the Stokes, as well as the Basset sub-problems. As you can see, trajectories are almost indistinguishable. Better overview on the difference of both sub-problems can be obtained from Fig.3., where the time evolution of difference of particle position components between the Basset and the Stokes problem relatively to the characteristic length (current loop radius $a$ ) are shown. Although the trajectories are indistinguishable, there is obvious delay of time evolution of particle position components for the Basset sub-problem in comparison with the Stokes one. 
Table 1. Values of geometrical and physical parameters used in simulations.

\begin{tabular}{|c|c|c|c|}
\hline Description & Symbol & Value & Unit \\
\hline \multicolumn{4}{|l|}{ Particle: $M y O n e^{T M}[38]$} \\
\hline Particle diameter & $2 b$ & $10^{-6}$ & $\mathrm{~m}$ \\
\hline Particle mass density & $\rho_{\mathrm{p}}$ & 1791 & $\mathrm{~kg} / \mathrm{m}^{3}$ \\
\hline Particle mag. susceptibility & $\chi$ & 1.433 & 1 \\
\hline \multicolumn{4}{|l|}{ Fluid ambient: air at $300 \mathrm{~K}$} \\
\hline Fluid mass density & $\rho_{\mathrm{f}}$ & 1.177 & $\mathrm{~kg} / \mathrm{m}^{3}$ \\
\hline Fluid dynamic viscosity & $\eta$ & $18.5 \times 10^{-6}$ & Pa.s \\
\hline \multicolumn{4}{|l|}{ a) Loop problem ${ }^{a}$} \\
\hline Loop radius & $a$ & $5.0 \times 10^{-3}$ & $\mathrm{~m}$ \\
\hline Loop current magnitude & $I$ & 5.0 & A \\
\hline Characteristic length ${ }^{b}$ & $a$ & $a$ & $\mathrm{~m}$ \\
\hline Characteristic velocity $^{b}$ & $U$ & calculated & $\mathrm{m} / \mathrm{s}$ \\
\hline Characteristic time $^{b}$ & $T$ & calculated & $\mathrm{s}$ \\
\hline \multicolumn{4}{|l|}{ b) Cyclotron problem ${ }^{c}$} \\
\hline Angular velocity & $\omega$ & $2 \pi \times 10^{5}$ & $\mathrm{rad} / \mathrm{s}$ \\
\hline Angular velocity vector & $\vec{\omega}$ & $(0,0, \omega)$ & $\mathrm{rad} / \mathrm{s}$ \\
\hline Initial radial distance & $a$ & $0.1 \times 10^{-3}$ & $\mathrm{~m}$ \\
\hline Characteristic length & $a$ & $a$ & $\mathrm{~m}$ \\
\hline Characteristic velocity & $U$ & $\omega a$ & $\mathrm{~m} / \mathrm{s}$ \\
\hline Characteristic time & $T$ & $2 \pi a / U$ & $\mathrm{~s}$ \\
\hline
\end{tabular}

${ }^{a}$ Magnetic particle moving in magnetic field of circular current loop.

${ }^{b}$ See B.

${ }^{c}$ Particle moving in cyclotron-like magnetic field.

\section{Stokes vs. Basset problem}

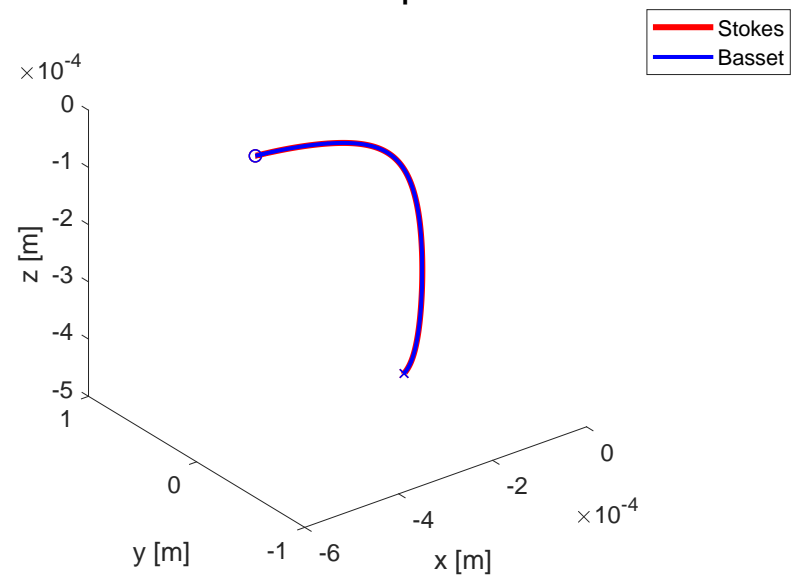

Fig. 2. (Color online.) Comparison of trajectories of magnetic particle in circular current loop magnetic field in air as quiescent fluid ambient solved as the Stokes and the Basset problem. Values of parameters used in simulations are shown in Table 1.

An overview on the dynamics of the Basset sub-problem can be obtained from the time evolution of each kind of force (their absolute values) acting on moving particle shown in Fig.4.; and their mean value averaged over the whole time of movement shown as histogram in Fig.5. Magnetic force drives the motion with major counteracting Stokes drag force.
The movement is also decelerated with the Basset history force, however, three orders of magnitude lower than the Stokes drag. Similar effect has added mass force term with five orders of magnitude lower contribution than the Basset force term. Buoyancy reduced gravity acts also as constant decelerating force in vertical direction for the given geometry of model.

Dominant effect of the Basset history force term is presented at an early stage of movement, as well as in the close vicinity of the circular current loop with the highest gradient of magnetic field and, therefore, the highest magnetic force (Fig.4.). The latter is the most significant for this problem.

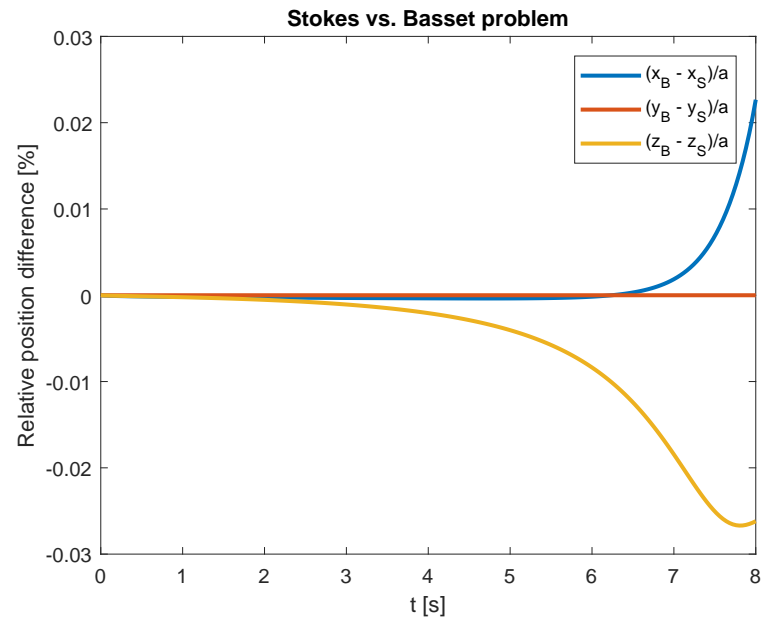

Fig.3. (Color online.) Time evolution of ratio of particle position (its components) difference for the Basset (subscript B) and the Stokes (subscript $\mathrm{S}$ ) sub-problem to the characteristic length (loop radius) for magnetic particle in the magnetic field of circular current loop. Values of parameters used in simulations are shown in Table 1.

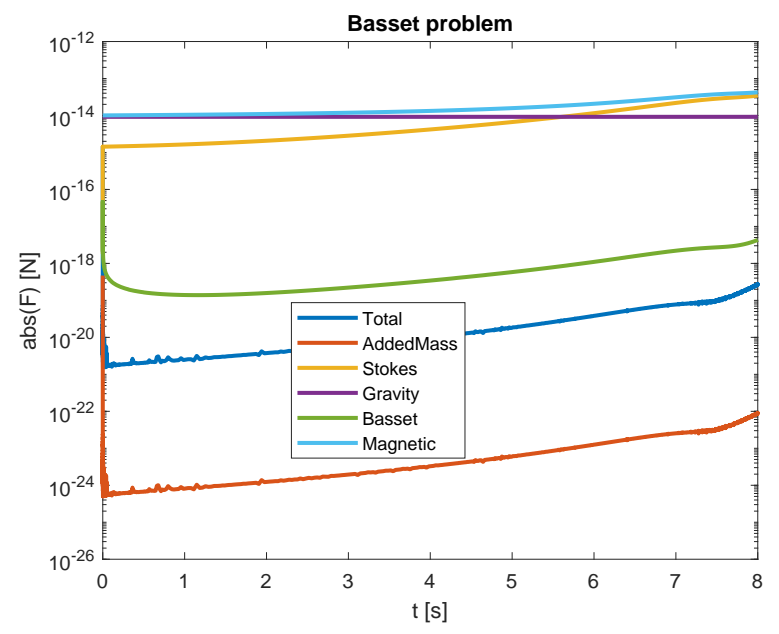

Fig. 4. (Color online.) Time evolution of absolute values of forces acting on moving particle in the magnetic field of circular current loop for the Basset problem. Values of parameters used in simulations are shown in Table 1. 


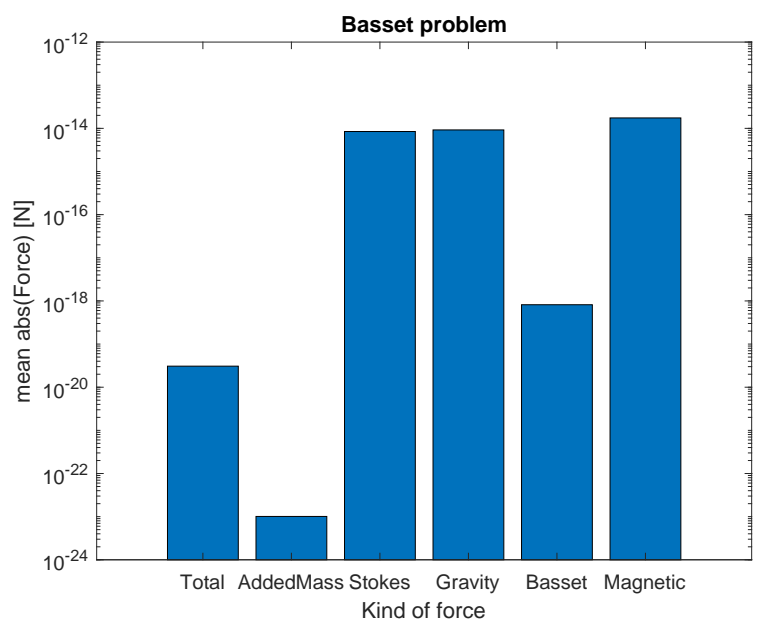

Fig. 5. (Color online.) Histogram of kinds of forces (their absolute values averaged over the whole time evolution) acting on moving magnetic particle in the magnetic field of circular current loop for the Basset problem. Values of parameters used in simulations are shown in Table 1.

\subsection{Particle in cyclotron-like homogeneous magnetic field}

In Fig.6. are shown trajectories of particle in the cyclotronlike homogeneous magnetic field in air as quiescent fluid ambient for both, the Stokes and the Basset sub-problems. The significant difference is obvious, as you can see also in Fig.7., where the time evolution of the ratio of the difference of particle position components between the Basset and the Stokes sub-problem and the characteristic length (particle initial radial distance $a$ ) are shown.

Insight to the dynamics and significance of each kind of force acting on the moving particle in the cyclotron-like magnetic field and quiescent air for the Basset sub-problem can be taken from the time evolution of $x$ - and $y$-components, and absolute value of each kind of force in Fig.8., and also from the histogram of mean values of forces averaged over the whole time evolution in Fig.9. The movement of particle is driven by magnetic force with almost exponential decay, influenced mainly with one-half order lower Stokes drag with similar time evolution. The Basset history force acts as decelerator with more complicated behavior and huge values at early stage of movement due to non-zero initial relative velocity of particle and quiescent air ambient [contrary to zero initial relative velocity in the case of magnetic particle moving in the field of circular current loop problem (Section 3.1)]. Added mass force term copies exponential decay behavior with huge values also at an early stage of movement. Constant buoyancy reduced gravity accelerates the movement of particle in vertical direction and together with viscous forces causes particle settling. Magnetic force and the Stokes drag have a major effect on particle movement averaged over the whole time evolution. The mean Basset history force term magnitude is lower about one and half order. The added mass force term is lower than the Basset term about another two orders. Buoyancy reduced gravity has the weakest contribution.

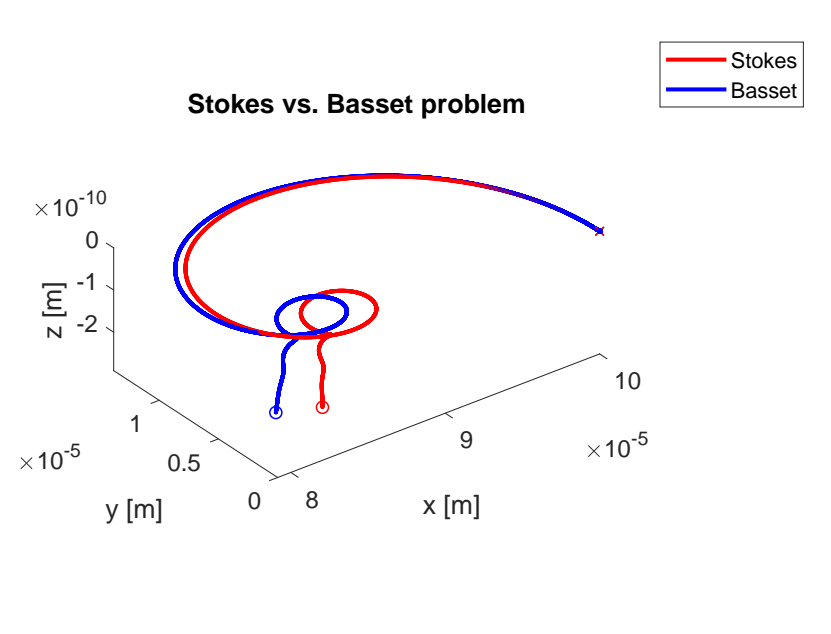

Fig. 6. (Color online.) Comparison of trajectories of moving particle in cyclotron-like homogeneous magnetic field in air as quiescent fluid ambient solved as the Stokes and the Basset problem. Values of parameters used in simulations are shown in Table 1.

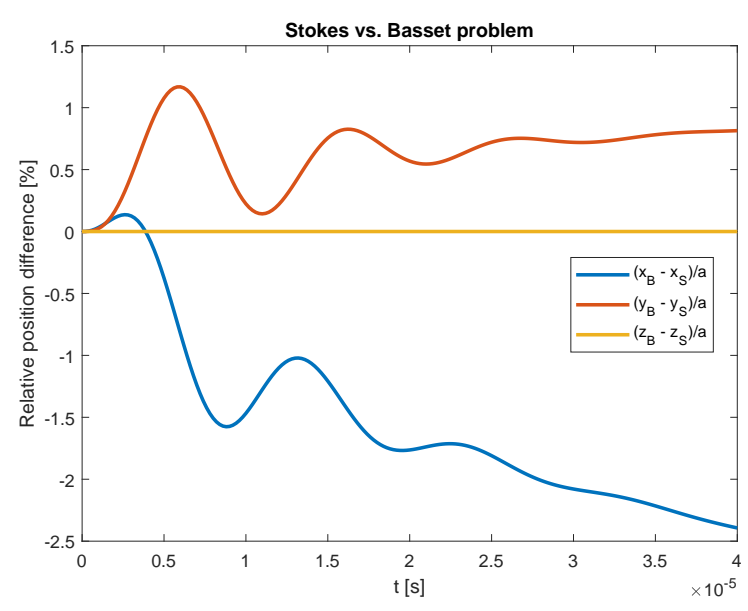

Fig. 7. (Color online.) Time evolution of the ratio of particle position (its components) difference for the Basset (subscript B) and the Stokes (subscript S) problem and the characteristic length (particle initial radial distance) for particle in the cyclotron-like homogeneous magnetic field. Values of parameters used in simulations are shown in Table 1.

As can be seen from Fig.8., $x$ - and $y$-component of total force is decaying exponentially with visible oscillations. Similar exponential decay is also present for magnetic and Stokes forces in the Stokes problem. Although, for the Basset problem their behavior is affected with memory effect of history force which causes oscillation of absolute value of velocity of the particle about values exponentially decaying in the Stokes problem (Fig.10.), which reflects inherent periodic circular motion in cyclotron-like magnetic field (Fig.6.). Magnetic and Stokes forces depend linearly on velocity, therefore small depressions appear with some delay also in their values. 
(a)

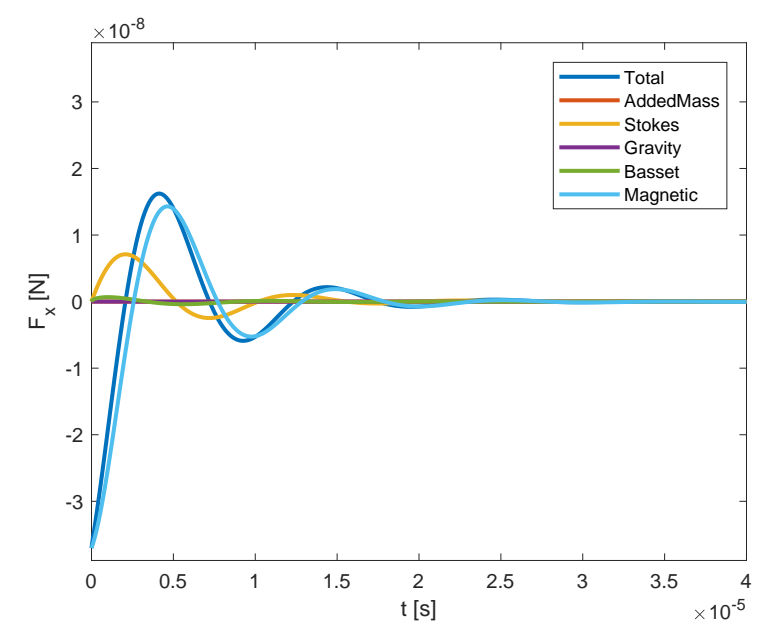

(b)

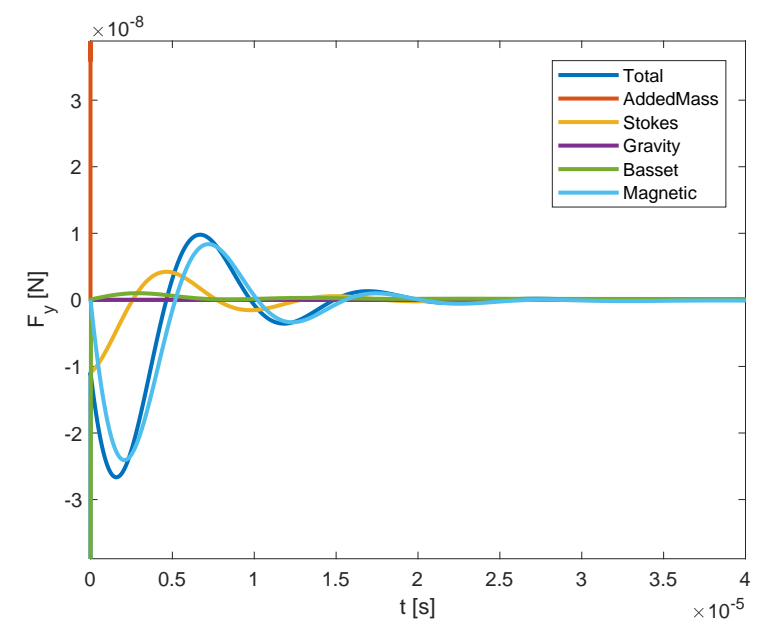

(c)

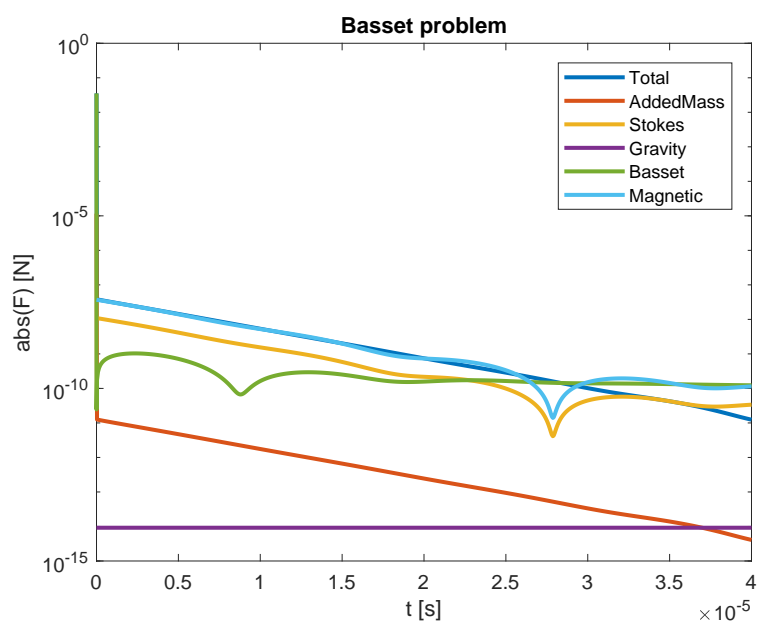

Fig. 8. (Color online.) Time evolution of (a) $x$-, (b) $y$-components and (c) absolute values of forces acting on moving particle in the cyclotron-like magnetic field for the Basset problem. Values of parameters used in simulations are shown in Table 1.

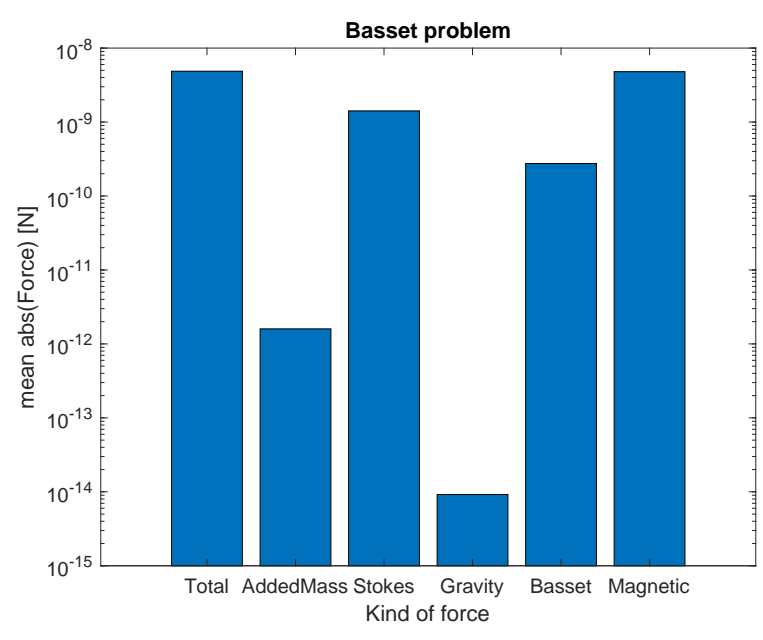

Fig.9. (Color online.) Histogram of kinds of forces (their absolute values averaged over the whole time evolution) acting on moving particle in the cyclotron-like magnetic field for the Basset problem. Values of parameters used in simulations are shown in Table 1.

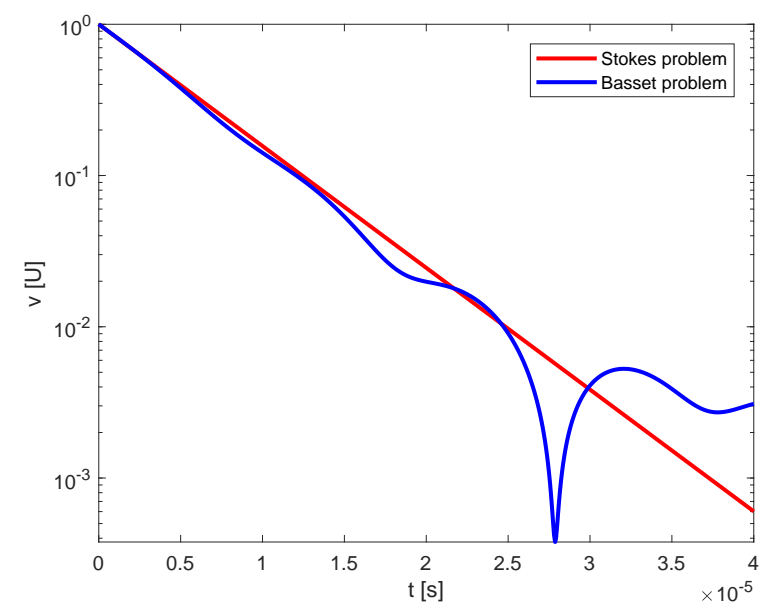

Fig. 10. (Color online.) Time evolution of absolute value of instantaneous particle velocity for Stokes and Basset problems, their comparison, for particle in cyclotron-like magnetic field. Values of parameters used in simulations are shown in Table 1.

\subsection{Relevance and validity conditions}

For relevant applications of the Maxey-Riley equation as the governing equation for the description of studied systems, several validity conditions have to be fulfilled [11], [32]. The first one, particle Reynolds number $R e_{\mathrm{p}} \equiv|\vec{v}-\vec{u}| b / v$ remaining small during the entire dynamics, is fulfilled for both simulated problems and each sub-problem (as you can see illustratively for the Basset sub-problem in Fig.11.). In addition, the size parameter $S$ must be small (i.e., the particle's characteristic time scale is much smaller than that of the flow), what is also fulfilled (see Table 2.; the size parameter $S$ is a more appropriate number to characterize memory effects than 
the traditional Stokes number, which is $S t \equiv S / R$ in our notation [11]). The last condition, which assures that the so-called Faxén corrections are negligible [32], is that the particle radius must be much smaller than the characteristic length scale of the flow: $b \ll a$, what is fulfilled.

Table 2. Characteristic values of scales and calculated dimensionless numbers.

\begin{tabular}{|c|c|c|c|c|}
\hline Parameter & \multicolumn{2}{|c|}{ a) Loop problem ${ }^{a}$} & \multicolumn{2}{|c|}{ b) Cyclotron problem ${ }^{b}$} \\
\hline \multicolumn{5}{|c|}{ Characteristic values of scales: } \\
\hline$a$ & $0.5 \times 10^{-3}$ & $\mathrm{~m}$ & $0.1 \times 10^{-3}$ & $\mathrm{~m}$ \\
\hline$U$ & $10.8 \times 10^{-3}$ & $\mathrm{~m} / \mathrm{s}$ & 62.8 & $\mathrm{~m} / \mathrm{s}$ \\
\hline$T$ & $46.3 \times 10^{-3}$ & $\mathrm{~s}$ & $10 \times 10^{-6}$ & s \\
\hline$v_{\mathrm{m}}$ & $0.11 \times 10^{-3}$ & $\mathrm{~m} / \mathrm{s}$ & 212.9 & $\mathrm{~m} / \mathrm{s}$ \\
\hline \multicolumn{5}{|c|}{ Dimensionless numbers: } \\
\hline$R$ & $0.66 \times 10^{-3}$ & & $0.66 \times 10^{-3}$ & \\
\hline$S$ & $76.5 \times 10^{-9}$ & & $0.35 \times 10^{-3}$ & \\
\hline$W$ & $-4.9 \times 10^{-3}$ & & $-0.84 \times 10^{-6}$ & \\
\hline$R e_{\mathrm{p}}^{c}$ & $\leq 6.3 \times 10^{-6}$ & & $\leq 2.0$ & \\
\hline$S t^{d}$ & $0.12 \times 10^{-3}$ & & 0.54 & \\
\hline
\end{tabular}

${ }^{a}$ Magnetic particle moving in magnetic field of circullar current loop.

${ }^{b}$ Particle moving in cyclotron-like magnetic field.

${ }^{c}$ Particle Reynolds number $R e_{\mathrm{p}}=|\vec{v}| b / v$.

${ }^{d}$ Stokes number $S t=S / R$.

\section{CONCLUSIONS}

The objective of this study was the analysis and implementation of a model to characterize the importance of various forces arising in the accelerated motion of magnetic particle in quiescent air as fluid ambient and in external high gradient magnetic field of circular current loop (the first modeled problem), as well as, of particle carrying charge in cyclotronlike homogeneous magnetic field (the second modeled problem). For the first modeled problem: even the trajectory of particle described with the Maxey-Riley equation is almost undistinguishable from the case considering only magnetic, gravitational and Stokes force; a significant delay of particle capturing is observed. Also for the second modeled problem the difference of trajectory of particle in cyclotron-like magnetic field is well distinguishable.

As we have found, at the relevant time-scales of the order from milliseconds to fraction of seconds, the Basset force cannot be neglected for a correct description of the studied systems, especially for the movement at the early stages, as well as in regions with the highest driving forces.

\section{A. Circular CURRENT LOOP MAGNetiC FluX DEN- SITY}

Single circular current loop magnetic flux density and its partial derivatives in the Cartesian coordinate system using elliptic integrals $K\left(k^{2}\right), E\left(k^{2}\right)$ [35]:

$$
B_{x}=\frac{C x z}{2 \alpha^{2} \beta \rho^{2}}\left(\left(a^{2}+r^{2}\right) E\left(k^{2}\right)-\alpha^{2} K\left(k^{2}\right)\right),
$$

$$
\begin{aligned}
B_{y} & =\frac{C y z}{2 \alpha^{2} \beta \rho^{2}}\left(\left(a^{2}+r^{2}\right) E\left(k^{2}\right)-\alpha^{2} K\left(k^{2}\right)\right)=\frac{y}{x} B_{x}, \\
B_{z} & =\frac{C}{2 \alpha^{2} \beta}\left(\left(a^{2}-r^{2}\right) E\left(k^{2}\right)+\alpha^{2} K\left(k^{2}\right)\right),
\end{aligned}
$$

where $C \equiv \mu_{0} I / \pi, \alpha^{2} \equiv a^{2}+r^{2}-2 a \rho, \beta^{2} \equiv a^{2}+r^{2}+2 a \rho$, $k^{2} \equiv 1-\alpha^{2} / \beta^{2}, \gamma \equiv x^{2}-y^{2}, \rho^{2} \equiv x^{2}+y^{2}, r^{2} \equiv \rho^{2}+z^{2}$; with $I$ and $a$ as the current and radius of circular current loop in $x y$-plane with axis identical with $z$-axis of the Cartesian coordinate system, respectively; and $\mu_{0}$ as the vacuum permeability.

$$
\begin{aligned}
& \frac{\partial B_{x}}{\partial x}=\frac{C z}{2 \alpha^{4} \beta^{3} \rho^{4}}\left\{\left[a^{4}\left(-\gamma\left(3 z^{2}+a^{2}\right)+\rho^{2}\left(8 x^{2}-y^{2}\right)\right)\right.\right. \\
& -a^{2}\left(\rho^{4}\left(5 x^{2}+y^{2}\right)-2 \rho^{2} z^{2}\left(2 x^{2}+y^{2}\right)+3 z^{4} \gamma\right) \\
& \left.-r^{4}\left(2 x^{4}+\gamma\left(y^{2}+z^{2}\right)\right)\right] E\left(k^{2}\right) \\
& +\left[a^{2}\left(\gamma\left(a^{2}+2 z^{2}\right)-\rho^{2}\left(3 x^{2}-2 y^{2}\right)\right)\right. \\
& \left.\left.+r^{2}\left(2 x^{4}+\gamma\left(y^{2}+z^{2}\right)\right)\right] \alpha^{2} K\left(k^{2}\right)\right\}, \\
& \frac{\partial B_{x}}{\partial y}=\frac{C x y z}{2 \alpha^{4} \beta^{3} \rho^{4}}\left\{\left[3 a^{4}\left(3 \rho^{2}-2 z^{2}\right)-r^{4}\left(2 r^{2}+\rho^{2}\right)\right.\right. \\
& \left.-2 a^{6}-2 a^{2}\left(2 \rho^{4}-\rho^{2} z^{2}+3 z^{4}\right)\right] E\left(k^{2}\right) \\
& +\left[r^{2}\left(2 r^{2}+\rho^{2}\right)-a^{2}\left(5 \rho^{2}-4 z^{2}\right)\right. \\
& \left.\left.+2 a^{4}\right] \alpha^{2} K\left(k^{2}\right)\right\}, \\
& \frac{\partial B_{x}}{\partial z}=\frac{C x}{2 \alpha^{4} \beta^{3} \rho^{2}}\left\{\left[\left(\rho^{2}-a^{2}\right)^{2}\left(\rho^{2}+a^{2}\right)\right.\right. \\
& \left.+2 z^{2}\left(a^{4}-6 a^{2} \rho^{2}+\rho^{4}\right)+z^{4}\left(a^{2}+\rho^{2}\right)\right] E\left(k^{2}\right) \\
& \left.-\left[\left(\rho^{2}-a^{2}\right)^{2}+z^{2}\left(\rho^{2}+a^{2}\right)\right] \alpha^{2} K\left(k^{2}\right)\right\}, \\
& \frac{\partial B_{y}}{\partial x}=\frac{\partial B_{x}}{\partial y}, \\
& \frac{\partial B_{y}}{\partial y}=\frac{C z}{2 \alpha^{4} \beta^{3} \rho^{4}}\left\{\left[a^{4}\left(\gamma\left(3 z^{2}+a^{2}\right)+\rho^{2}\left(8 y^{2}-x^{2}\right)\right)\right.\right. \\
& -a^{2}\left(\rho^{4}\left(5 y^{2}+x^{2}\right)-2 \rho^{2} z^{2}\left(2 y^{2}+x^{2}\right)-3 z^{4} \gamma\right) \\
& \left.-r^{4}\left(2 y^{4}-\gamma\left(x^{2}+z^{2}\right)\right)\right] E\left(k^{2}\right) \\
& +\left[a^{2}\left(-\gamma\left(a^{2}+2 z^{2}\right)-\rho^{2}\left(3 y^{2}-2 x^{2}\right)\right)\right. \\
& \left.\left.+r^{2}\left(2 y^{4}-\gamma\left(x^{2}+z^{2}\right)\right)\right] \alpha^{2} K\left(k^{2}\right)\right\}, \\
& \frac{\partial B_{y}}{\partial z}=\frac{y}{x} \frac{\partial B_{x}}{\partial z}, \\
& \frac{\partial B_{z}}{\partial x}=\frac{\partial B_{x}}{\partial z} \\
& \frac{\partial B_{z}}{\partial y}=\frac{\partial B_{y}}{\partial z}, \\
& \frac{\partial B_{z}}{\partial z}=\frac{C z}{2 \alpha^{4} \beta^{3}}\left\{\left[6 a^{2}\left(\rho^{2}-z^{2}\right)-7 a^{4}+r^{4}\right] E\left(k^{2}\right)\right. \\
& \left.+\alpha^{2}\left(a^{2}-r^{2}\right) K\left(k^{2}\right)\right\} \text {. }
\end{aligned}
$$

For paraxial limit case see the original source [35]. 
(a)

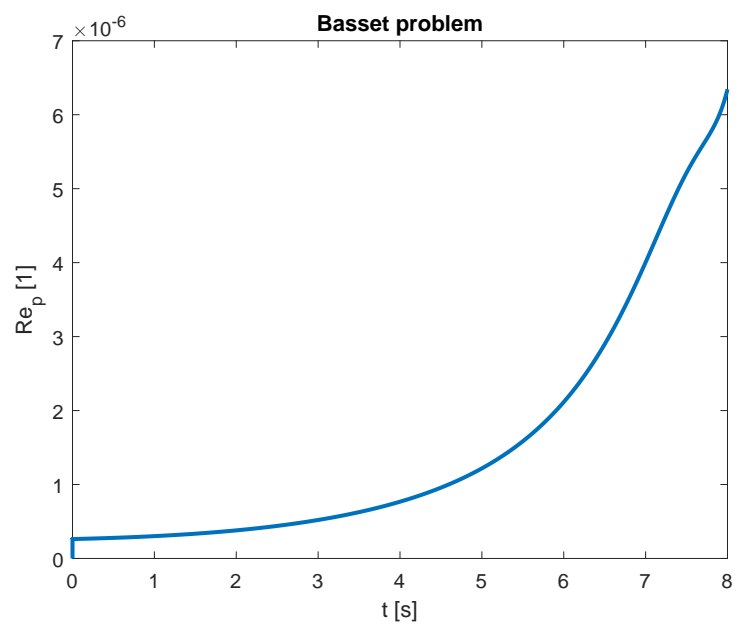

(b)

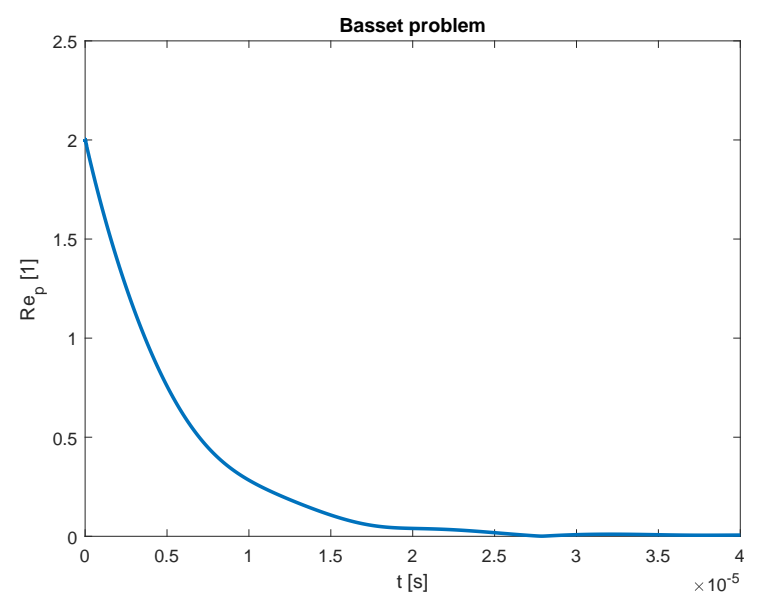

Fig.11. (Color online.) Time evolution of the particle Reynolds number of (a) magnetic particle moving in magnetic field of a circular current loop; and (b) particle moving in cyclotron-like magnetic field in air as quiescent fluid ambient for the Basset problem. Values of parameters used in simulation are shown in Table 1.

\section{B. CIRCULAR CURRENT LOOP PROBLEM SCALING}

For magnetic particle in gradient magnetic field of circular current loop problem we have evaluated values of characteristic parameters (length, velocity and time) numerically, solving the movement of magnetic particle from the center of circular current loop to its rim in the plane of circular current loop in vacuum and gravity-free condition. Explicitly solving an initial value problem:

$$
m_{\mathrm{p}} \frac{\mathrm{d} \vec{v}}{\mathrm{~d} t}=\vec{F}_{\mathrm{Mag}}, \quad \vec{r}(0)=\overrightarrow{0}, \quad \vec{v}(0)=\overrightarrow{0},
$$

where the magnetic force is defined as (7) with magnetic flux density $\vec{B}$ and its derivative specified in A. In this way, the characteristic length $a$ is defined as a radius of the circular current loop. The characteristic time $T$ is defined as a time of movement from the center to the rim of the circular current loop. And finally, the characteristic velocity norm as a $U \equiv$ $a / T$.

\section{ACKNOWLEDGEMENTS}

This work was supported by the Slovak Scientific Grant Agency, projects VEGA 2/0003/20 and 1/0810/18, and within the project of the Slovak Research and Development Agency Nr. APVV-15-0029 and APVV-16-0600.

\section{REFERENCES}

[1] Golozar, M., Molki, M., Darabi, J. (2017). Computational and performance analysis of a continuous magnetophoretic bioseparation chip with alternating magnetic fields. Microfluid. Nanofluid. 21(4), 73.

[2] Gomez-Pastora, J., Karampelas, I. H., Xue, X., Bringas, E., Furlani, E. P., Ortiz, I. (2017)a. Magnetic bead separation from flowing blood in a two-phase continuous- flow magnetophoretic microdevice: Theoretical analysis through computational fluid dynamics simulation. $J$. Phys. Chem. C 121(13), 7466-7477.

[3] Gomez-Pastora, J., Xue, X., Karampelas, I., Bringas, E., Furlani, E. P., Ortiz, I. (2017)b. Analysis of separators for magnetic beads recovery: From large systems to multifunctional microdevices. Sep. Purif. Technol. 172, 16-31.

[4] Babinec, P., Krafcik, A., Babincova, M., Rosenecker, J. (2010). Dynamics of magnetic particles in cylindrical Halbach array: Implications for magnetic cell separation and drug targeting. Med. Biol. Eng. Comput. 48(8), 745-753.

[5] Krafcik, A., Babinec, P., Babincova, M. (2010). Feasibility of subcutaneously implanted magnetic microarrays for site specific drug and gene targeting. J. Eng. Sci. Technol. Rev. 3(1), 53-57.

[6] Durdik, S., Krafcik, A., Babincova, M., Babinec, P. (2013). Conceptual design of integrated microfluidic system for magnetic cell separation, electroporation, and transfection. Phys. Med. 29(5), 562-567.

[7] Krafcik, A., Babinec, P., Frollo, I. (2014). Computational analysis of magnetic field induced deposition of magnetic particles in lung alveolus in comparison to deposition produced with viscous drag and gravitational force. J. Magn. Magn. Mater. 380, 46-53.

[8] Basset, A. B. (1888). A Treatise on Hydrodynamics. Cambridge: Deighton, Bell and Co.

[9] Daitche, A., Tel, T. (2011). Memory effects are relevant for chaotic advection of inertial particles. Phys. Rev. Lett. 107(24), 244501.

[10] Guseva, K., Feudel, U., Tel, T. (2013). Influence of the history force on inertial particle advection: Gravitational effects and horizontal diffusion. Phys. Rev. E Stat. Nonlinear Soft Matter Phys. 88(4), 042909. 
[11] Daitche, A., Tel, T. (2014). Memory effects in chaotic advection of inertial particles. New J. Phys. 16, 073008.

[12] Elghannay, H. A., Tafti, D. K. (2016). Development and validation of a reduced order history force model. Int. J. Multiph. Flow 85, 284-297.

[13] Guseva, K., Daitche, A., Feudel, U., Tel, T. (2016). History effects in the sedimentation of light aerosols in turbulence: The case of marine snow. Phys. Rev. Fluids $1(7)$.

[14] van Hinsberg, M. A. T., Clercx, H. J. H., Toschi, F. (2017). Enhanced settling of nonheavy inertial particles in homogeneous isotropic turbulence: The role of the pressure gradient and the Basset history force. Phys. Rev. E 95(2), 023106.

[15] Thomas, P. J. (1992). On the influence of the Basset history force on the motion of a particle through a fluid. Phys. Fluids A 4(9), 2090-2093.

[16] Abbad, M., Souhar, M. (2004). Effects of the history force on an oscillating rigid sphere at low Reynolds number. Exp. Fluids. 36(5), 775-782.

[17] Garbin, V., Dollet, B., Overvelde, M., Cojoc, D., Di Fabrizio, E., van Wijngaarden, L., Prosperetti, A., de Jong, N., Lohse, D., Versluis, M. (2009). History force on coated microbubbles propelled by ultrasound. Phys. Fluids 21(9), 092003.

[18] Mei, R., Klausner, J. F., Lawrence, C. J. (1994). A note on the history force on a spherical bubble at finite Reynolds number. Phys. Fluids 6(1), 418-420.

[19] Klinkenberg, J., De Lange, H. C., Brandt, L. (2014). Linear stability of particle laden flows: The influence of added mass, fluid acceleration and Basset history force. Meccanica 49(4), 811-827.

[20] Olivieri, S., Picano, F., Sardina, G., Iudicone, D., Brandt, L. (2014). The effect of the Basset history force on particle clustering in homogeneous and isotropic turbulence. Phys. Fluids 26(4), 041704.

[21] Krafcik, A., Babinec, P., Babincova, M., Frollo, I. (2019). High gradient magnetic separation with involved Basset history force: Configuration with single axial wire. Powder Technol. 347, 50-58.

[22] McKee, S., Stokes, A. (1983). Product integration methods for the nonlinear Basset equation. SIAM Journal on Numerical Analysis 20(1), 143-160.

[23] Dorgan, A. J., Loth, E. (2007). Efficient calculation of the history force at finite Reynolds numbers. Int. J. Multiph. Flow 33(8), 833-848.

[24] van Hinsberg, M. A. T., ten Thije Boonkkamp, J. H. M., Clercx, H. J. H. (2011). An efficient, second order method for the approximation of the Basset history force. J. Comput. Phys. 230(4), 1465-1478.

[25] Daitche, A. (2013). Advection of inertial particles in the presence of the history force: Higher order numerical schemes. J. Comput. Phys. 254, 93-106.

[26] Moreno-Casas, P. A., Bombardelli, F. A. (2016). Computation of the Basset force: Recent advances and en- vironmental flow applications. Environ. Fluid Mechan. 16(1), 193-208.

[27] von Kriegstein, E., von Kriegstein, K. (2007). Inhaled insulin for diabetes mellitus. New Engl. J. Med. 356(20), 2106-2108.

[28] Dames, P., Gleich, B., Flemmer, A., Hajek, K., Seidl, N., Wiekhorst, F., Eberbeck, D., Bittmann, I., Bergemann, C., Weyh, T., Trahms, L., Rosenecker, J., Rudolph, C. (2007). Targeted delivery of magnetic aerosol droplets to the lung. Nat. Nanotechnol. 2(8), 495-499.

[29] Laurent, S., Saei, A. A., Behzadi, S., Panahifar, A., Mahmoudi, M. (2014). Superparamagnetic iron oxide nanoparticles for delivery of therapeutic agents: Opportunities and challenges. Expert Opin. Drug Deliv. 11(9), 1449-1470.

[30] Price, D. N., Stromberg, L. R., Kunda, N. K., Muttil, P. (2017). In vivo pulmonary delivery and magnetictargeting of dry powder nano-in-microparticles. Mol. Pharm. 14(12), 4741-4750.

[31] Russo, F., Boghi, A., Gori, F. (2018). Numerical simulation of magnetic nano drug targeting in patient-specific lower respiratory tract. J. Magn. Magn. Mater. 451, 554-564.

[32] Maxey, M. R., Riley, J. J. (1983). Equation of motion for a small rigid sphere in a nonuniform flow. Physics of Fluids 26(4), 883-889.

[33] Michaelides, E. E. (1992). A novel way of computing the Basset term in unsteady multiphase flow computations. Phys. Fluids A 4(7), 1579-1582.

[34] Zborowski, M., Chalmers, J. J. (eds.) (2008). Magnetic Cell Separation, Vol. 32 of Laboratory techniques in biochemistry and molecular biology. Elsevier.

[35] Simpson, J. C., Lane, J. E., Immer, C., Youngquist, R. C. (2001). Simple analytic expressions for the magnetic field of a circular current loop. Preprint, NTRS-NASA Technical Reports Server. URL: https://ntrs.nasa.gov/archive/nasa/casi. ntrs.nasa.gov/20010038494.pdf accessed: 30 June 2017.

[36] Krafcik, A., Babinec, P., Frollo, I. (2018). Stokes versus Basset: Comparison of forces governing motion of small bodies with high acceleration. Eur. J. Phys. 39(3), 035805 .

[37] Shampine, L. F., Reichelt, M. W. (1997). The MATLAB ode suite. SIAM J. Sci. Comput. 18(1), 1-22.

[38] ThermoFischer Scientific (2018). Dynabeads ${ }^{\mathrm{TM}}$ MyOne $^{\mathrm{TM}}$. Technical report, ThermoFischer Scientific. URL: https://www.thermofisher.com/order/ catalog/product $/ 65012$ ? SID =srch-srp -65012 accessed: 31 May 2018.

Received October 16, 2019. Accepted March 2, 2020. 